\title{
Wheat-alien introgression breeding: current status and prospects in the 21st century
}

\author{
Adonina I.G.*, Salina E.A. \\ Institute of Cytology and Genetics, SB RAS, Novosibirsk, Russia \\ *e-mail: adonina@bionet.nsc.ru
}

Wheat-alien introgression breeding went through several stages. The first attempts to obtain hybrids between wheat and related species have been carried out at the end of the 19th century. Initially, the purpose of the experiments was to study the evolution and origin of species, the meiotic chromosome pairing. However, the main purpose of distant hybridization was to create wheat with exceptional properties. It seemed that this approach would provide much wider possibilities for enriching the wheat genome than traditional selection. Introgression breeding reached its peak in the second half of the 20th century. A significant progress has been made in developing strategies to produce hybrids of wheat with distant relatives, in the improvement of cytogenetic techniques, development of molecular markers to identify and characterize introgressed chromatin. These advances led to development of a large panel of introgression lines of various types and from a number of wild wheat relatives, carrying important traits. At present, based on the accumulated data, one can speak about certain regularities of distant hybridization of wheat. For example, in chromosomes 4A, 5A, the least alien introgression is observed. On the contrary, it is possible to distinguish "hot" sites on individual chromosomes and whole chromosomes, where introgression occurs more often. Nowadays, only a small number of commercially successful wheat cultivars have been created by distant hybridization. The potential of alien introgression breeding remains underused. What are the prospects for wheat-alien introgression breeding in the 21 st century? Primarily, we need to develop theoretical knowledge. Almost nothing is known how the wheat genome interacts with introgressed genes and how it influences their function. The recent advances in genomics, transcriptomics, epigenomics, proteomics, in cytogenetics are promising to deliver the needed insights. The use of the new plant breeding techniques can be useful. In this respect, cis-genesis and genome editing, in particular based on CRISPR/Cas9, are promising.

Acknowledgements: The study has been supported by the Budget project 0324-20190039. 